\section{HER2 als Prognosefaktor bei Magenkrebs}

\author{
Eine Überexpression des humanen epidermalen Wachstumsfaktors 2 (HER2) \\ kommt nicht nur bei Brustkrebs vor, sondern auch bei verschiedenen anderen \\ epithelialen Krebsarten, z.B. bei Tumoren in Darm, Magen oder Lunge. Ob die \\ HER2-Expression das Überleben von Patienten mit Magenkarzinom beein- \\ flusst, wollten australische Chirurgen wissen.
}

ine systematische Übersichtsarbeit
sollte klären, welche Bedeutung
HER2 für das Überleben von Patienten
mit Magenkarzinom hat und ob ein Zu-
sammenhang mit klinisch-pathologischen
Eigenschaften des Tumors besteht. 49
Studien mit insgesamt 11.337 Patienten
wurden in die Analyse aufgenommen.
$44 \%$ der Patienten hatten ein Magenkar-
zinom in den Stadien I-II, $56 \%$ in den
Stadien III-IV. Bei $18 \%$ der Magenkarzi-
nome war eine HER2-Überexpression
vorhanden, zu deren Bestimmung meist
immunhistochemische Tests verwendet
wurden.
In sechs Studien wurde der Einfluss der
HER2-Expression auf das krankheitsfreie
Überleben untersucht. Dabei ergab sich
entweder kein Unterschied (drei Studien) oder ein signifikant schlechteres Überleben bei HER2-Überexpression (drei Studien). Die krankheitsfreie 3-Jahres-Überlebensrate betrug bei Patienten mit HER2Überexpression $58 \%$ und bei Patienten ohne HER2-Überexpression $86 \%$ (vier Studien).

Der Einfluss der HER2-Überexpression auf das Gesamtüberleben wurde in 35 Studien untersucht. In 20 dieser Studien ergab sich kein Unterschied im Gesamtüberleben, in zwei Studien hatten Patienten mit HER2-Überexpression ein signifikant besseres Gesamtüberleben und in 13 Studien ein signifikant schlechteres Gesamtüberleben. Das mediane Gesamtüberleben betrug bei Patienten mit HER2Überexpression 21 Monate und bei Patienten ohne HER2-Überexpression 33
Monate (27 Studien). Die 5-Jahres-Überlebensraten betrugen $42 \%$ bzw. $52 \%$.

Außerdem scheint eine positive Assoziation zwischen der HER2-Überexpression und Karzinomen vom intestinalen Typ zu bestehen. Dies war die einzige Korrelation zu klinisch-pathologischen Eigenschaften. Eine Aussage über einen Zusammenhang zwischen der HER2Expression und anderen klinisch-pathologischen Eigenschaften wie WHO-Klassifikation, Tumorstadium, Lymphknotenbefall und Metastasen war nicht möglich, weil die verschiedenen Studien widersprüchliche Ergebnisse lieferten.

Fazit: Nach der aktuellen Studienlage ist davon auszugehen, dass Patienten mit Magenkrebs, bei denen HER2 überexprimiert ist, schlechtere Überlebenschancen haben als Patienten ohne HER2-Überexpression.

Judith Neumaier

Chua TC, Merrett ND. Clinicopathologic factors associated with HER2-positive gastric cancer and its impact on survival outcomes A systematic review. Int J Cancer. 2012;130(12):2845-56.

\title{
Magenkarzinom: Lymphknotenstation 7 als Besonderheit
}

Nicht nur das Ausmaß der Lymphknotenbeteiligung sondern auch anatomische Besonderheiten wie etwa der ausschließliche Befall der Station 7 (entlang der Arteria gastrica sinistra) spielen für die Prognose des Magenkarzinoms eine Rolle.

$\mathrm{n}$ ihrer Analyse werteten die Autoren Fälle von 1.606 Patienten mit Magenkarzinom aus, die in der Histologie einen Befall der Lymphknoten gezeigt hatten. Bei ihnen war neben der Resektion eine D2- oder D3-Lymphadenektomie durchgeführt worden. Bei allen Studienteilnehmern war der Schnittrand tumorfrei.

In Gruppe A ( $\mathrm{n}=734$ Patienten) lag eine Metastasierung von Lymphknoten des Levels I gemäß der Japanischen Klassifikation von Magenkarzinomen (JCGC, 13. Version) vor, in Gruppe B ( $\mathrm{n}=317$ Patienten) Metastasen in Lymphknoten des
Levels II, in der ausschließlich Lymphknoten der Station 7 entlang der Arteria gastrica sinistra befallen waren. In Gruppe C $(n=501)$ waren Studienteilnehmer mit Metastasierung auf Level II ohne Befall der Lymphknotenstation 7 zusammengefasst, in Gruppe D $(n=54)$ Patienten mit Metastasen auf Level III.

In Gruppe B waren zwar mehr Lymphknoten befallen als in Gruppe A - Tumorgröße und Infiltration (pT-Stadium) waren in beiden Gruppen A und B jedoch vergleichbar. Die 5-Jahres-Gesamtüberlebensrate in Gruppe B lag mit 41,7\% etwas niedriger als in Gruppe A (48,0\%, $\mathrm{p}=0,038$ ). Patienten der Gruppe $C$ wiesen nur eine Überlebensrate von 27,6\% auf, der Unterschied gegenüber Gruppe B war ebenfalls signifikant $(\mathrm{p}<0,001)$.

Die Autoren führten mehrere Vergleichsanalysen durch, bei denen die Anzahl befallener Lymphknoten (TNM-System des American Joint Committee on
Cancer) sowie das Verhältnis von befallenen zu untersuchten Lymphknoten berücksichtigt wurde. Dabei zeigten sich vergleichbare Überlebenskurven ohne signifikante Unterschiede zwischen Gruppe A und B. Patienten der Gruppe C wiesen auch hier eine schlechtere Prognose auf.

Fazit: Patienten mit Befall von Lymphknoten des Levels II nach JCGC - und zwar ausschließlich der Lymphknoten entlang der Arteria gastrica sinistra - hatten bei gleicher Anzahl positiver Lymphknoten eine ähnliche Prognose wie die mit Befall von Lymphknoten des Levels I. Die Autoren empfehlen, Tumoren mit Befall dieser speziellen Lymphknotengruppe als Nodalstadium I zu klassifizieren. Andreas Fischer

Tong JH et al. Prognostic significance of lymph node station 7 for patients with gastric cancers underwent radical surgery.

J Surg Oncol. 2012;105(8):805-12. 